\title{
Phase retrieval in the focal plane
}

\author{
W. Gaessler ${ }^{1, a}$, D. Peter ${ }^{1}$, and C. Storz ${ }^{1}$ \\ Max-Planck-Institut für Astronomie \\ Königstuhl 17 \\ 69117 Heidelberg \\ Germany
}

\begin{abstract}
Phase retrieval in the focal plane is a very appealing technique, which would simplify the optomechanics of an Adaptive Optics (AO) system a lot and could gain sensitivity under certain conditions. For conventional AO systems the limiting magnitude of the system does not depend on the diameter of the telescope, since any wave front sensor splits the light into sub-apertures, which are in number related to the telescope diameter. Having this in mind the phase retrieval technique looks promising as it breaks this paradigm in the diffraction limited case and thus yields some gain in limiting magnitude with larger telescope diameter. Until now this path was not followed deeply in astronomical AO systems, as the solution of the inversion is non unique and demands much higher calculation power as in conventional AO. This might change with state of the art computers. We give a short overview of some existing techniques and algorithms of focal plane AO and report results of other groups, which tested them in laboratory and on sky. To solve the drawback of the large computational demands and to increase the sensitivity we propose a bootstrapping process with dynamical binning.
\end{abstract}

\section{Introduction}

Current Adaptive Optics (AO) systems, which are using natural guide stars (NGS), are limited by the guide star's magnitude needed to achieve a proper signal to noise ratio for an appropriate performance. Such limitation is overcome by laser guide stars (LGS) but payed with increase in complexity and overhead. This is especially true in the case of wide field AO, which requires multiple guide stars. Science case analysis for Extremely Large Telescope (ELT) projects show that wide field AO on one side and high contrast AO on the other will be the two most demanded modes for new breakthroughs in astronomy [1], [2] and [3]. In most of the ELT projects the implementation of the AO system work with Shack-Hartmann wavefront sensors locked on multiple LGS [4], [5]. Such solution needs still technology development in large and fast detector arrays and reliable high power laser systems - apart from large diameter deformable mirrors.

Complex systems are much more susceptible to failure and demand much more maintenance. Therefore, it is worth to look further for a more simple solution. The simplest way would be to have no wavefront sensor at all but use the science detector directly for wavefront sensing. A method is needed to retrieve the phase from the focal plane. Such methods are already around since the seventies of the last century [10], [6]. Also the technical implementation is available now. Recently we implemented a new parallel sub-window readout into our MPIA IR-readout electronic. Such readout allows non destructive reads of sub windows, i.e. illuminated with stars, during the science exposure, which could be used for wavefront sensing. The electronic does also work with the old HAWAII 2 detectors and allow for a retrofit with reduced performance. Apart from the possibility and the simplicity of the sensor another question arises. Could one gain in limiting magnitude, as the fix splitting of light in sub-apertures of the usual wavefront sensors seems not to be done?

In the next chapter we will introduce the problem of FPAO, than we will discuss the sensitivity problem. After that we describe methods how to implement FPAO. Finally we conclude with a summary and some thoughts how we think FPAO could/should be facilitated.

\footnotetext{
${ }^{a}$ e-mail: gaessler@mpia.de
} 


\section{Focal Plane AO (FPAO)}

A typical and simple imaging system is shown in Fig. 1. The distorted wavefront at the pupil plane can be described with a function $U_{i}$. The intensity of the image on the focal plane can be described as:

$$
I=\left|U_{f}\right|^{2}
$$

where $U_{f}$ is the Fourier Transform of $U_{i}$.

$$
U_{f}=F\left(U_{i}\right)
$$

To retrieve the wavefront on the pupil plane from the intensity distribution in the focal plane one has to solve:

$$
I=\left|F\left(U_{i}\right)\right|^{2}
$$

Such equation has non unique solutions and is non linear. The usual retrieval procedures are iterative, which are quite calculation intensive. But state of the art computers should be able to handle such problem.

The advantage for such kind of AO are more on the optomechanical part as it is a simple setup, there is no need of additional parts. The measurement is as close as possible to the science image and one has not to deal with non-common path abberations.

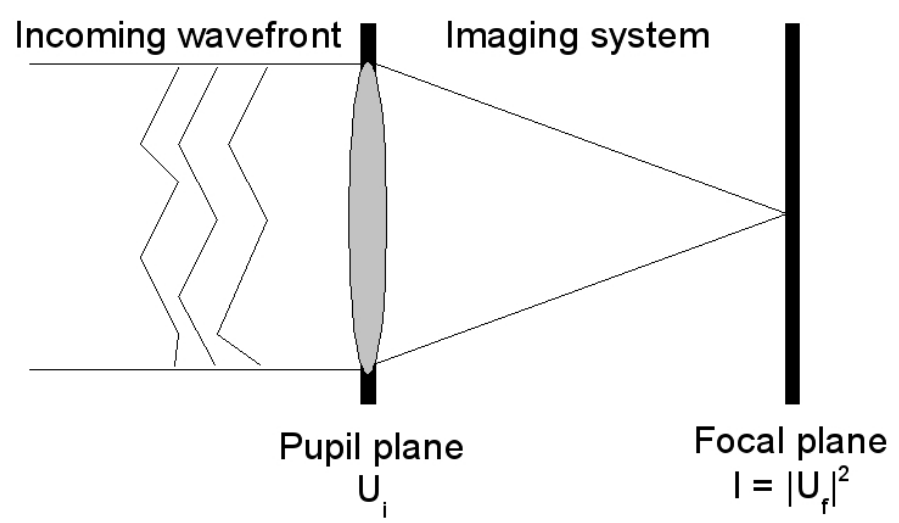

Fig. 1. Most simple imaging system as one could use for FPAO to illustrate the problem.

\section{Sensitivity}

Larger telescopes are built to collect more photons and to observe fainter objects. The number of photons for the same object scales with the collection area of the telescope and is proportional to $D^{2}$. Adaptive optics using NGS is limited by the brightness of its guide star. But larger telescopes do not provide fainter guide stars as the number of sub-apertures needed to calculate the correction do also scale with $D^{2}$. Therefore, a larger telescope diameter has no gain in sensitivity for AO with NGS. 
This changes for the Tip/Tilt measurements of a LGS system, which is still done with a NGS. As the number of modes and with that the number of sub-apertueres for the Tip/Tilt measurement is fix, the number of photons scales with the diameter squared over the number of sub-apertures and sampling.

In the focal plane the sensitivity depends on the sampling of the PSF. If the pixel sampling follows the diameter of the telescope, the number of photons per pixel scales with the diameter squared and the efficency even with the power of four, in the diffraction limited case. However, in the seeing limited case the PSF is oversampled and the photons are spread in $\left(D / r_{0}\right)^{2}$ more pixels. This means: FPAO can increase the sensitivity only in the diffraction limited case. How can one get to this condition and already making some use of the larger telescope diameter? More photons from the same object are delivered by a larger telescope. Only the sampling is to be adjusted to the current condition. In the seeing limited case one needs a coarser sampling than in the diffraction limited case. Such dynamical binning can be done either with software binning, where one looses sensitivity because of the readout noise for each pixel, which multiply, or with hardware binning where one always can adjust to the optimal gain proportional to $D^{2}$.

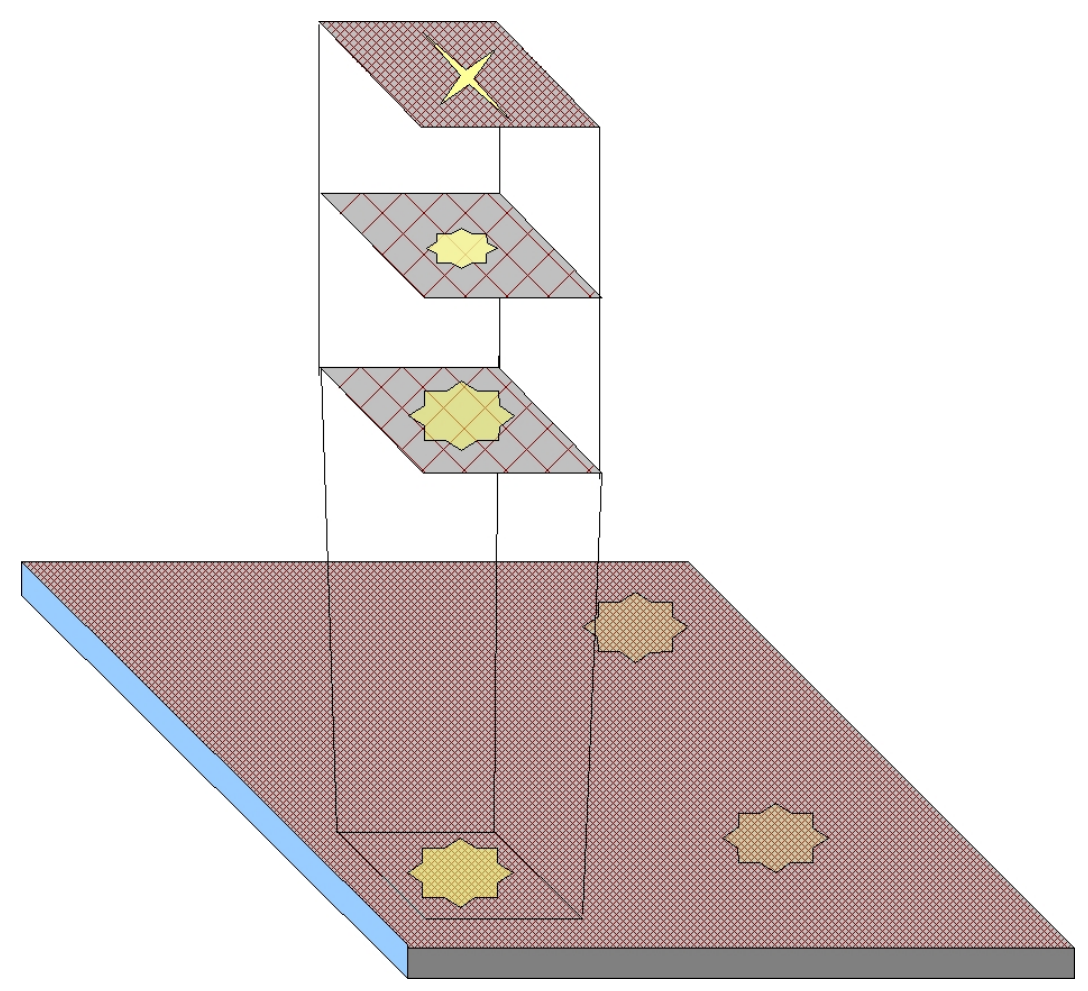

Fig. 2. Illustration of the dynamical binning during the bootstrap.

\section{Methods of FPAO}

Two principal methods could be found, which are applicable to FPAO:

- Image sharpening

- Iterative Fourier Transform

The first method, image sharpening, was already proposed for astronomy in the 70's of the last century [6] and soon after tested on sky with a multi mirror telescope [7]. Recently such algorithms have been improved and extended for systems with large degrees of freedom [8], [9]. 
The iterative Fourier Transform method is based on the Gerchberg-Saxton algorithm [10], which are available in various implementations.

\subsection{Image sharpening}

The method of image sharpening is based on the optimization of a given metric. The advantages and disadvantages of several metricizes are discussed extensively in [6]. The most promising metricizes are summarized here:

$$
\text { Maximize }: S=\int I^{n}(x, y) d x d y \quad \text { with } \mathrm{n}=2,3,4
$$

where $I(x, y)$ is the irradiance at the position $\mathrm{x}$ and $\mathrm{y}$. The metric defines an aberrated image as less than the true image and has its optimum when all abberations are corrected. This kind of metric can also be used at extended objects. Power law metrics with small power perform better when the object has no prominent scatter. The next metric minimizes the entropy of the image:

$$
\text { Maximize }: S=-\int I(x, y) \ln (|I(x, y)|) d x d y
$$

again with $I(x, y)$ as the irradiance at the position $\mathrm{x}$ and $\mathrm{y}$. The metric minimizes the higher-order moments of the distribution. But it is only proven for low wavefront errors. A more promising metric, which extend the approach to larger aberrations is proposed by [8]:

$$
\text { Minimize }:<\rho^{2}>=\left(\frac{\lambda}{N A}\right)^{2}|\mathbf{b}|^{2}
$$

where $\rho$ is the spot radius, NA is the size of the numerical aperture, $\lambda$ the wavelength and $\mathbf{b}$ is a set of Lukosz-Zernike function. The Lukosz-Zernike function are polynomial, which express the wavefront in a radial and an azimuthal way but relate more direct to the spot radius as this is the optimization metric.

All these metricizes reach iteratively their optimum by introducing different trial phase corrections, i.e. with the deformable mirror. As the number of possibilities scale with the actuator dynamic powered by the number of actuators the convergence can take quite long for high order correction. Murray et. al. [9] proposed schemes to reduce this to $N+1$ iterations, where $N$ is the number of actuators. Multiplexing as proposed for some AO calibration schemes [11] might reduce the number of iterations further.

\subsection{Iterative inverse Fourier Transform}

The iterative inverse Fourier transform method is based on the Gerchberg-Saxton algorithm. The wavefront in the pupil plane with an approximation of amplitude as constant is inverse Fourier transformed into the image plane. The result is compared with the PSF in the image plane and Fourier transformed into the pupil plane. By variation of the phase the comparison is minimized in an iterative way. Fig. 3 illustrates the process.

The calculation of such algorithm is quite computation power intensive and scales with the number of modes and pixels. However, using a bootstrapping process and starting with large binning and reducing the binning with each step accelerates the process as already tested by O. Guyon [12].

\section{Conclusion}

Phase retrieval in the focal plane is a long known problem worked on with several solutions: 


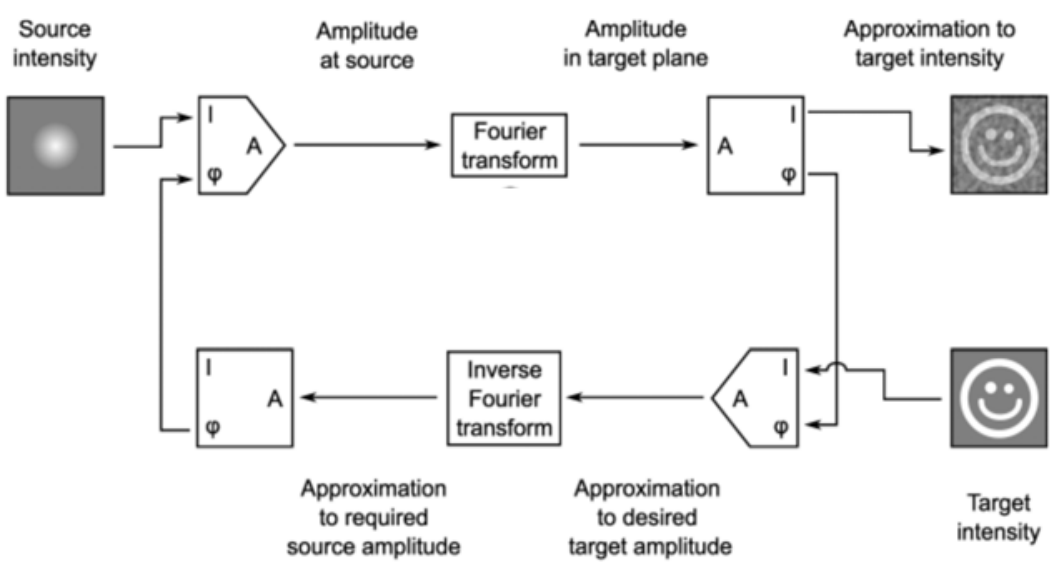

Fig. 3. Illustration of the iterative process of the Gerchberg-Saxton algorithm. The picture is taken and modified from wikipedia (http://en.wikipedia.org/wiki/Gerchberg-Saxton_algorithm).

- Image sharpening

- Iterative Inverse Fourier transform

The drawback of large computational needs and iterations can be overcome with dynamical binning, in the case of the iterative inverse Fourier transform. Dynamic binning in a bootstrap process can accelerate the computation and increase sensitivity for NGS. How fast such process can become is still to be evaluated in detail. But nevertheless one can already use such method for truth sensing in the case of LGS or compensation of slow changing non-common path errors, where speed is not such a big issue.

\section{References}

1. M. Kissler-Patig, this proceeding, 2009

2. J. Nelson, this proceeding, 2009

3. P. McCarthy, this proceeding, 2009

4. N. Hubin, this proceeding, 2009

5. B. Ellerbroek, this proceeding, 2009

6. R. A. Muller, JOSA 64/9, 1974, p1201

7. A. Buffington, JOSA 67/3, 1977, p304

8. M. J. Booth, SPIE 6711, 2007

M. J. Booth, SPIE 6306, 2006

M. J. Booth, OptExp 14/4, 2006, p1340

M. J. Booth, OptLet 32/1, 2006, p5

9. L.P. Murray, SPIE 5823, 2005, p40

L.P. Murray, SPIE 6018, 2005

10. R. W. Gerchberg and W. O. Saxton, Optik 35, 1972, p237

11. S. Oberti, SPIE 6272, 2006

12. O. Guyon, privat communication 